Página inicial: 127 - Página final: 145

TIPO DE ARTÍCULO: de Investigación

\title{
DIAGNÓSTICO PSICOSOCIAL DE UN BARRIO EN LA CIUDAD DE ARMENIA ${ }^{1}$.
}

\author{
PSYCHO-SOCIAL DIAGNOSIS OF A NEIGHBORHOOD IN THE CITY OF ARIENIA.
}

\author{
Recibido: Junio 2013 Revisado: Octubre 2013 Aceptado: Diciembre 10 de 2013 \\ Por: Maria Catalina Echeverri Londoño', Julia Raquel Meneses Ariza ${ }^{2}$ \\ Maria Cristina Restrepo Sierra $^{3}$ y Paula Andrea Marin Pava ${ }^{4}$.
}

\section{RESUMEN.}

Este artículo presenta los resultados un diagnostico psicosocial realizado a un barrio de la ciudad de Armenia, en el cual se identificaron las principales problemáticas que vive esta comunidad: robos, expendio y consumo de sustancias psicoactivas y múltiples manifestaciones de violencia urbana que evidencian el débil capital social existente. Para llevar a cabo este diagnóstico, se realizaron talleres y entrevistas a niños, niñas, jóvenes y adultos del barrio, árbol de problemas y cartografia social. Se evidenció un cambio en la percepción de la comunidad sobre la violencia e inseguridad en el barrio, que indica un supuesto descenso en la ocurrencia de estas problemáticas. Al realizar una discusión retomando teorias que abordan el tema de la violencia y las drogas, se infirió que las bandas dedicadas al control y expendio de drogas en el barrio han pasado de una delincuencia desorganizada a una organizada, lo cual explica la aparente disminución de las problemáticas. Asimismo, se realizó un contraste de resultados a la luz de la teoría del capital social y el bienestar social, en el que se sugirieron posibles estrategias de intervención para que la comunidad participe activamente en la resolución de sus dificultades.

\section{PALABRAS CLAVES.}

Diagnostico psicosocial, delincuencia, drogas, violencia, capital social, bienestar social.

\begin{abstract}
.
This article presents the results of a psycho-social diagnosis performed to a neighborhood of the city of Armenia, in which the main problems affecting this community were identified: thefts, sale and consumption of psychoactive substances and multiple manifestations of urban violence that underscore the weak existing social capital. To carry out this diagnosis, workshops and interviews with children, young people and adults of the neighborhood, tree problems and social mapping were carried out. A change in the perception of the community on violence and insecurity in the area was made evident, indicating a supposed decline in the occurrence of these problems. By carrying out a discussion and taking up theories that address the issue of violence and drugs, it was inferred that gangs dedicated to the control and sale of drugs in the neighborhood have moved from a disorganized to an organized crime which explains the apparent decline of the problems. In the same way, a contrast of results was made in the light of the theory of the social capital and social welfare, in which possible intervention strategies were suggested so that the community may actively participate in the resolution of their difficulties.
\end{abstract}

\section{KEY WORDS.}

Psycho-social Diagnosis; Delinquency; Crime; Drugs; Violence, Social Capital; and Social Welfare.

\footnotetext{
${ }^{1}$ Psicóloga Pontificia Universidad Javeriana-Cali, candidata a Magister en Sociología Universidad del Valle. Docente investigadora Universidad de San Buenaventura Medellín extensión Armenia. Perteneciente al grupo de investigación estudios Clínicos y sociales en psicología. Colombia. mariacatalinaecheverri@hotmail.com.

${ }^{2}$ Licenciada en ciencias de la educación Universidad Pedagógica nacional, Magister en estructuras y procesos de aprendizaje universidad externado de Colombia. Docente investigadora Universidad de San Buenaventura Medellín extensión armenia Perteneciente al grupo de investigación estudios Clínicos y sociales en psicología. Colombia. Jmeneses1@hotmail.com .

${ }^{3}$ Estudiante de psicología joven investigadora, Universidad de San Buenaventura Medellín extensión Armenia. Colombia. mariacristinalila@hotmail.com

4 Joven unvestigadora, Universidad de San Buenaventura Medellín extensión Armenia. Colombia. Paulaandreamarin22@hotmail.com
} 


\section{Introducción.}

Este diagnóstico psicosocial es el producto de una investigación realizada en un barrio de la ciudad de Armenia por docentes y estudiantes del programa de psicología de la Universidad de San Buenaventura Medellín extensión Armenia. Este ejercicio parte de una preocupación sobre la situación social del sector, la cual ha estado enmarcada por la ocurrencia de hechos delictivos durante los últimos años: robos, expendio de sustancias psicoactivas y múltiples manifestaciones de violencia urbana, como asesinatos, amenazas y riñas.

Al respecto, en un taller realizado con niños y niñas del barrio, éstos manifestaron: "Roban mucho en el barrio, roban y matan mucho a las personas"; por su parte, algunos adultos mayores afirmaron que: "Hay mucha drogadicción, se reúnen en las esquinas a meter" (Taller con adultos mayores). Una multiplicidad de sucesos violentos que han suscitado la especulación y estigmatización de la situación social del barrio por parte de los medios de comunicación y la sociedad en general, evidenció la necesidad de realizar un diagnóstico psicosocial que visibilizara la situación real de esta comunidad, en un intento por dilucidar tanto sus problemáticas como sus factores protectores.

Desde esta perspectiva, se hace necesario describir la historia y características estructurales del barrio. Este se construyó en un proceso de reubicación luego del terremoto ocurrido el 25 de enero de 1999 y que afectó gran parte del territorio del Quindio, especialmente la ciudad de Armenia. Este proceso fue adelantado por la ONG Federación Nacional de Vivienda Popular (FENAVP) en el año 2.000. Inicialmente, el proyecto contempló la construcción de ocho manzanas, dos zonas verdes y varias calles de acceso; no obstante, sólo se construyeron tres manzanas y una calle principal. De acuerdo con lo descrito por algunos habitantes, en este proceso de reubicación llegaron a vivir allí no sólo personas que estaban en situación de vulnerabilidad tras haber perdido sus viviendas, sino otras que residian en distintos barrios de la ciudad y municipios del país, que no contaban con vivienda propia y vieron en esta circunstancia una oportunidad para beneficiarse.

Cabe resaltar que los múltiples hechos delictivos y violentos que han ocurrido en los últimos años han llamado la atención de varias entidades públicas y privadas, las cuales han realizado caracterizaciones del barrio y ejecutado diferentes programas sociales orientados a mitigar tales problemáticas. Sin embargo, la mayoría de estas intervenciones han sido de corta duración y no han tenido continuidad, por lo cual no se han conformado redes comunitarias cohesionadas y empoderadas; asimismo, la poca e itinerante presencia de diversas isntituciones ha influido en que la comunidad no reconozca la legitimidad de estos procesos ni sienta confianza en ellos.

Todas las razones anteriormente expuestas fueron suficientes para tomar la decisión de adelantar un diagnostico psicosocial que permitiera indagar más allá de los aspectos demográficos de esta comunidad (Aguilar \& Ander-Egg, 2001). Las caracterizaciones realizadas anteriormente aportaron datos de corte sociodemográfico y estadístico sobre los aspectos económicos, laborales, de calidad de vivienda y problemáticas de salud de su población. Así, la presente investigación pretendió conocer de forma más exhaustiva cómo son percibidas tales problemáticas por parte de los habitantes y qué elementos del contexto y la cultura han precipitado su aparición. 
Se consideró necesario dar un paso más allá de la caracterización estadística y demográfica, puesto que los habitantes, más que objetos de estudio, son seres humanos que viven en unas condiciones sociales derivadas de la mala planificación urbana, del avance y mantenimiento del negocio de la droga y el delito, y de un sistema cultural particular que ha generado malestar en su vida privada y familiar. Sólo alrededor de este hecho cobra sentido lo expuesto por Blanco \& Varela (2007), quienes señalaron que parte de la justificación y razón de ser de las ciencias sociales, pero sobretodo de la psicología, es la construcción de bienestar, la apuesta por la búsqueda consciente de la solución a las necesidades y problemas, sean éstos individuales o sociales.

El propósito de la investigación fue elaborar un diagnóstico psicosocial y familiar de un barrio de la ciudad de Armenia, que permitiera visualizar y comprender de manera conjunta las principales problemáticas y recursos psicosociales, así como los elementos que inciden en la aparición de éstos. A partir de alli, se buscó identificar los procesos de organización y participación comunitaria de los habitantes del barrio; caracterizar las diferentes instituciones presentes y finalmente, encontrar puntos comunes y prioritarios de intervención en esta comunidad. Es importante aclarar que en este artículo se abordarán únicamente los resultados de corte sociocomunitario, más no los resultados de tipo familiar.

\section{Metodologia.}

El presente diagnóstico psicosocial se basó en un modelo de diseño mixto; en esta medida, desde el enfoque cuantitativo, se aplicaron instrumentos diagnósticos que permitieron identificar y describir las diferentes problemáticas; y desde lo cualitativo y de manera yuxtapuesta, se partió del discurso y los conocimientos de los habitantes del barrio a manera de investigación - acción participativa, técnica que parte de la consideración del objeto a investigar como protagonista de la investigación y cuya finalidad es la transformación social (Alberrich, 2007).

En un primer momento, se hizo necesario realizar un contacto previo con la comunidad, con la cual se realizaron diferentes tipos de campañas de socialización y sensibilización, a través de jornadas recreativas, talleres y charlas para padres de familia y jóvenes, y talleres con líderes comunitarios, en los que se recolectó información. Además se elaboraron los instrumentos, las categorías de análisis y la fundamentación teórica pertinente.

En el segundo momento, se llevó a cabo la recolección de información y el trabajo de campo, para lo cual se realizó un acercamiento a la población perteneciente a la institución educativa del barrio, a los habitantes de las diferentes manzanas y a los líderes comunitarios a través de la realización de talleres participativos, grupos de discusión, elaboración de cartografia social, árbol de problemas y entrevistas, sobre temas considerados como importantes por la comunidad, con el fin de iniciar la recolección de información concerniente a los objetivos propuestos.

Finalmente, en la tercera fase se realizó el análisis de los datos, para lo cual fue importante asegurar tanto la triangulación como la saturación de la información. Durante esta fase también se socializaron los resultados con la comunidad del barrio, la comunidad académica, y los entes estatales. 


\section{Resultados y discusión.}

Las problemáticas que se identificaron en este diagnóstico psicosocial están relacionadas con condiciones sociales y geográficas particulares del barrio, las cuales quedaron retratadas en la cartografia social (Ver Figura 1). En este sentido, cabe mencionar que una de las causas estructurales que influyó en la generación de las múltiples dificultades que se presentaron en la comunidad, fue la deficiente planeación con la que éste se construyó, ya que en un primer momento, se contemplaba la realización de ocho manzanas, dos zonas verdes y varias calles principales de acceso de las que terminaron siendo construidas sólo tres manzanas y una calle principal que sirve de acceso y salida al barrio. Dentro de la ejecución de la construcción tampoco se concretaron zonas verdes y de recreación; ante esta insuficiencia, los habitantes tomaron por su cuenta terrenos baldios para improvisar la construcción de canchas e implementar cultivos de hortalizas.

\section{Figura 1. Cartografia social}

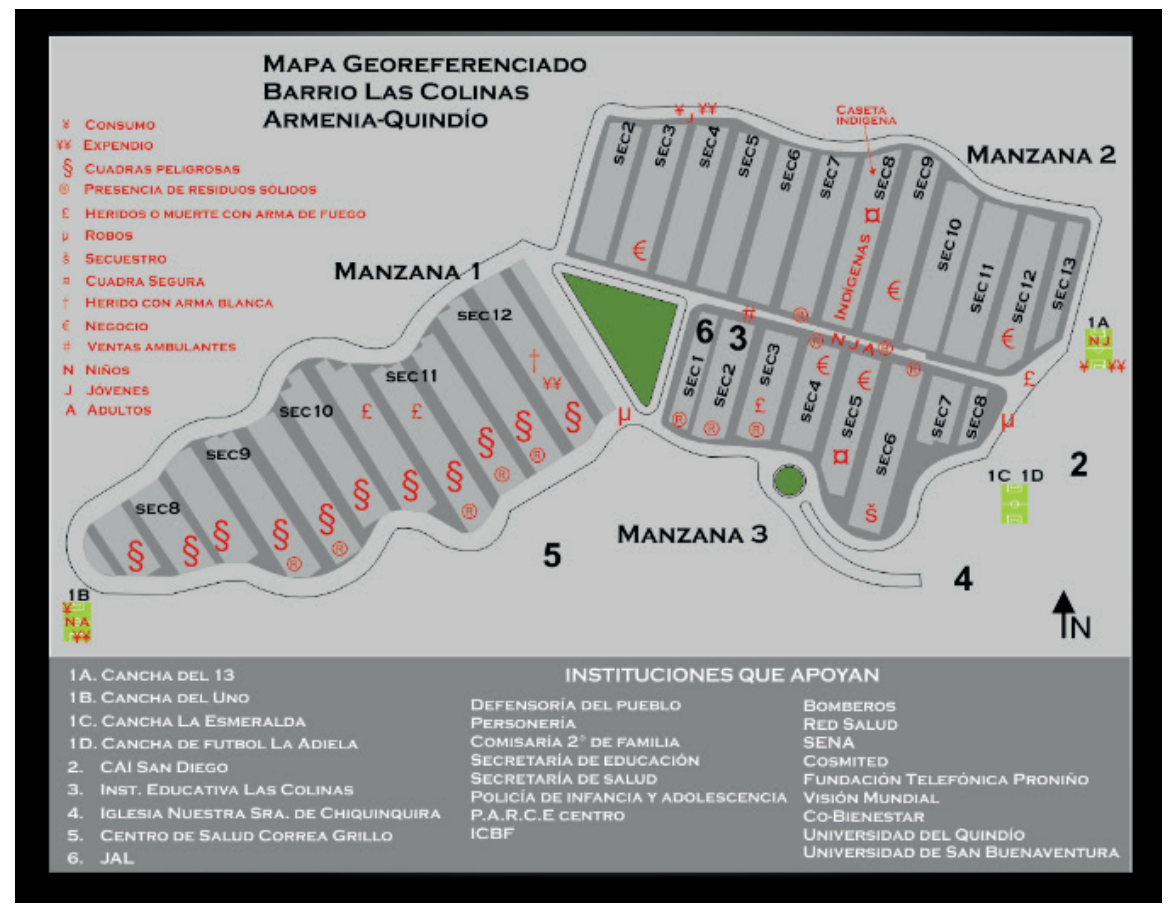

En cuanto a las condiciones geográficas y de movilidad, las calles se encuentran pavimentadas en su totalidad, y el acceso al transporte público es relativamente fácil, puesto que existe una ruta de buses que entra y sale del barrio por la calle principal; sin embargo, la disposición de ésta, que corresponde a un tramo inclinado, ha sido aprovechada por algunos habitantes para asaltar, hurtar pertenencias y cobrar "vacunas" a los conductores de buses de servicio público, tal como reporta una noticia del diario El Tiempo en la que se refiere que una de las pruebas que ponen a los jóvenes para ingresar a "combos" es atracar un bus que entre al barrio. (Sepúlveda, 2014, 28 de abril)

Cabe resaltar que al realizar el mapa para la cartografia social se hizo evidente la escasa presencia de instituciones estatales y no estatales, siendo la institución 
educativa; la caseta de la Junta de Acción Comunal; las iglesia cristianas y las guarderias del ICBF, las únicas instituciones con presencia física constante en el sector, y que de alguna manera están relacionadas con la generación de capital social y redes sociales en el barrio.

En relación con la escasa presencia estatal e institucional, es importante mencionar que el barrio no cuenta con un centro de salud que permita atender las necesidades de promoción de la salud y/o prevención de enfermedades, lo cual obliga a la comunidad a asistir a centros de salud de barrios aledaños. El barrio tampoco cuenta con una estación de policía, a pesar de la recurrencia de actos que afectan la seguridad ciudadana y la convivencia; las solicitudes que los habitantes han hecho en reiteradas ocasiones han sido respondidas a través de la presencia de la misma en rondas por cuadrantes durante el dia; sin embargo, la comunidad señala de forma generalizada que este ente gubernamental es ineficaz, en tanto no permanece en el barrio, ni en zonas de mayor ocurrencia de hechos delictivos. A continuación, la percepción de una habitante: "Al barrio le hace falta más seguridad, la policía está hasta al principio de la noche y a las 9 ya van saliendo" (Entrevista a habitante del barrio).

En cuanto a las instituciones que hacen presencia física y constante en el barrio, cabe mencionar a la institución educativa, la cual juega un papel primordial en la comunidad, ya que en torno a ésta se desarrollan valiosos procesos de construcción de capital social, no sólo por su labor académica, sino porque a su infraestructura se vinculan otros agentes que proveen apoyo económico, cultural, social y educativo de vital importancia para los habitantes; de ello dan testimonio principalmente los niños, que lo ven como un lugar agradable y de protección.

Pese a lo anterior, en un momento de la investigación fue posible encontrar otras perspectivas de los habitantes jóvenes y adultos, que relacionan al colegio como un lugar que empieza a perder su potencial de cohesión social y de alternativa para la recreación y el uso adecuado del tiempo libre, y empieza a ser referenciado como un lugar en el que también ocurren situaciones de inseguridad, como la registrada por el diario La Crónica (Meneses, 2013, 3 de octubre):

La Institución Educativa Las Colinas canceló las clases de esta semana. La decisión fue tomada porque los alumnos no quisieron hacer presencia en el plantel durante martes y miércoles por temor a que se cumplieran amenazas que se han presentado en los últimos dias y por una violenta situación que se registró en el barrio el pasado lunes, cuando al parecer hubo disparos y lanzaron una granada que no estalló.

Por otra parte, frente a la Junta de Acción Comunal (JAC), algunos habitantes del barrio reconocen su presencia a través de algunos de sus representantes, y de las labores que desarrollan, las cuales catalogan como de suma importancia para atender necesidades del barrio. Sin embargo, algunos de los habitantes entrevistados hacen mención a lo que denominan poca comunicación de las actividades, razón por la cual no se enteran de lo que dicha entidad adelanta en el barrio; al respecto una habitante refirió: "Uno a veces pasa los tiempos y no se da cuenta de lo que la junta hizo" (Entrevista a habitante del barrio).

A partir de lo anterior, se pudieron identificar las ubicaciones específicas de las problemáticas encontradas en el barrio; así, las manzanas 1 y 2 , y de esta última los sectores 9 a 13, son las que más se ven afectadas por el expendio y consumo 
de drogas, y por algunas manifestaciones de violencia, como las peleas entre sus habitantes. Lo anterior puede deberse a que en estas manzanas están ubicadas dos canchas en las que se concentran individuos involucrados con estas actividades, espacios que se sitúan en los extremos del barrio, cuentan con poca iluminación y además, colindan con terrenos baldíos que están alejados de las viviendas. Este hecho es confirmado por un habitante del barrio, quien relató: "Esa cancha es punto aparte, porque es un sitio muy peligroso, allá por ejemplo, yo ni voy porque le tengo respetos de entrar por allá, eso se ven como le comente ahora personas de una imagen aterradora y da mucho pesar y hasta niños muy jóvenes y la mayoría en las drogas, es más, los mismos policias les da miedo meterse por allá" (Entrevista a habitante del barrio).

No obstante, existen zonas del barrio con dinámicas diferentes, como la calle principal, donde se asienta el comercio. Éste se compone principalmente de tiendas de abarrotes, cafés internet, droguerías y puestos de venta ambulante con los que algunas familias del barrio sustentan sus ingresos económicos. Es también en esta calle donde transita la mayor parte de vehículos de transporte público y privado que llegan al barrio. Desafortunadamente, este factor también hace de la calle principal el lugar en el que ocurren el mayor número de robos, los cuales son dirigidos principalmente a este tipo de transporte público y a personas foráneas.

Otra dificultad que vale la pena mencionar es el manejo de residuos sólidos por parte de los habitantes, ya que en esta calle se encuentra uno de los más grandes focos de basura acumulada, lo cual resulta preocupante, dado que es por esta vía por donde el carro recolector transita dos veces a la semana. Esta situación parece afectar particularmente a los sectores de la manzana 2 y 3 , que son los aledaños a la calle principal.

La presencia de cúmulos de basura esparcidos sobre la calle y los andenes de diferentes sectores es una evidencia latente de esta problemática. Como consecuencia de ello, se ha afectado considerablemente la salubridad en el barrio, dado que en los últimos años se han presentado varios casos de enfermedades producidas por vectores, como infecciones digestivas, respiratorias y en el peor de los casos, el dengue, a tal punto de causar la muerte a algunos habitantes. Al respecto, La Crónica del Quindío informó: Se encontró que la población del barrio Las Colinas de Armenia desarrolló inmunidad al virus del dengue, muchas personas tuvieron la enfermedad, pero eso los hace más vulnerables a que exista otro serotipo circulante o a una reinfección siendo más fácil que se presente el dengue hemorrágico. (La Crónica del Quindio, 2010, 26 de octubre).

Los resultados de la investigación lograron evidenciar también factores protectores, como la presencia de algunas instituciones y programas que se desarrollan con los habitantes del barrio; entre éstos se encuentran subsidios que otorga el gobierno y programas de psicoeducación que adelantan algunas ONG. Estos son, en su mayoría, programas intermitentes, lo cual hace dificil la consolidación de capital social de alta densidad.

También se destaca la presencia de un grupo de indígenas yanacona, que trabajan por su comunidad a través de grupos infantiles y juveniles, cuyo objetivo es la transmisión y conservación de sus valores culturales, y brindar una alternativa para que ocupen adecuadamente su tiempo, que les lleve a elegir opciones diferentes 
a las ofrecidas en el sector, que son la pertenencia a pandillas, el consumo de sustancias psicoactivas, la prostitución, entre otras.

En el proceso de identificación de las problemáticas de las cuales se viene hablando, en el curso de la investigación se realizó un hallazgo que está relacionado con la aparición de tres elementos problemáticos mencionados por la comunidad, que no se encontraban en las caracterizaciones que realizaron previamente otras instituciones; éstos son: las amenazas a diferentes personas del barrio, la presencia de lugares de expendio y de manera especial, la presencia en el barrio de bandas dedicadas al expendio de droga o microtráfico.

Son estos tres elementos los que parecen estar evidenciando un encrudecimiento de la situación de violencia que se vive en el barrio, y además, empiezan a evidenciar el escaso capital social que está presente allí.

La relación entre capital social, violencia y vulnerabilidad en barrios de ciudades latinoamericanas ha sido objeto de investigación en varias oportunidades, tal es el caso del estudio de Lunecke \& Ruíz (2006), quienes aseveran que en estos barrios se pueden identificar dos líneas de relación entre el capital social y la violencia: una linea donde la violencia y la delincuencia producen el debilitamiento del capital social comunitario, y otra en la que los mismos mecanismos utilizados por personas o grupos para construir capital social pueden derivar consecuencias negativas. Estamos, por lo tanto, ante la presencia de una reducción del capital social comunitario y frente al fortalecimiento de un capital social perverso, que se cristaliza en la violencia asociada a las pandillas y el tráfico de drogas.

Hasta el momento se han intentado describir algunas de las problemáticas y situarlas geográficamente en el sector, sin embargo, además de ello, durante todo el transcurso de la investigación se realizó un proceso de análisis e interpretación de los datos recogidos en el barrio, en aras de llegar a elementos comprensivos y sobre todo, a aquellos focos fundamentales de intervención que permitan iniciar un proceso de transformación social. Así, pues, en el siguiente paso se expondrán algunos puntos pertinentes de dicho análisis:

Desempleo, pobreza, prostitución y robo.

Uno de los elementos en los que se profundizó en la presente investigación fue el aspecto económico de sus habitantes; en este sentido, se indagó por las posibilidades de empleo y la satisfacción de necesidades básicas. De acuerdo con la información proporcionada por varios grupos etarios, se pudo determinar que en el barrio hay presencia tanto de empleo formal e informal, como de desempleo y pobreza, que en ciertas ocasiones llega a la pobreza extrema.

En algunos testimonios, es posible percibir que hay reconocimiento de que la comunidad es trabajadora. "Hay familias muy buenas, trabajadoras todas, porque todo el mundo no es malo acá. El 90 \% es trabajador", relató una habitante del barrio en una entrevista. Cabe anotar que entre los trabajos que desempeñan, están el de vendedores en negocios formales e informales que están ubicados en el barrio o en otras zonas de la ciudad y obreros de construcción, entre otros. Una habitante también comentó: "Por la mañana son esos buses llenos porque toda la gente se va a trabajar" (Entrevista a habitante del barrio), lo cual indica que la dinámica de muchas familias del sector está relacionada con el empleo. 
Cabe resaltar que el hecho de que haya una proporción considerable de habitantes trabajando en actividades distintas al robo y el microtráfico es un valioso recurso de la comunidad, una realidad que se ve subestimada ante el estereotipo que existe frente al barrio, que desafortunadamente termina incluyendo a la totalidad de sus habitantes.

No obstante, algunas de ellos afirmaron que hay familias que no cuentan con un sustento económico para satisfacer sus necesidades básicas, como lo señala un habitante del barrio: "hay mucha pobreza por falta de empleo" (tomado de taller con adultos). En este sentido, es importante recalcar que en el imaginario social de la comunidad se suele realizar una asociación entre desempleo, pobreza, prostitución, robo y consumo de sustancias, condiciones que constituyen un círculo de interrelación.

Frente a la prostitución, algunos habitantes del barrio refieren que hay personas que ejercen esta actividad porque están desempleadas y no tienen dinero para alimentar a sus familias, y también señalan que otras lo hacen para poder comprar drogas y alcohol. En cuanto a los robos, se pudo observar que la percepción que tiene la comunidad sobre este tema ha cambiado, puesto que algunos habitantes afirman que en años pasados se veían más casos en el barrio, pero que en los últimos dos años los robos a viviendas de este sector han disminuido. Esto responde a que últimamente han involucrado como víctimas a personas que no residen en el barrio, es decir, conductores de buses de transporte público, de taxis y/o personas que llegan en vehículos particulares.

Al respecto, se pudo indagar también que quienes roban agreden a las personas físicamente, causándoles en algunas ocasiones heridas con arma blanca o de fuego. Estos hechos ocurren en partes estratégicas, donde los vehículos deben parar o avanzar despacio, principalmente en los alrededores del sector 1 de la manzana 1 y sobre la calle principal.

Con todo lo anterior, se puede inferir que la comunidad encuentra una relación entre el robo, pobreza, inseguridad y violencia, que a su vez están estrechamente relacionados tanto con el expendio como con el consumo de sustancias psicoactivas. Esta interrelación ha acarreado una ambigüedad en los discursos de los habitantes, quienes exponen diferentes versiones sobre tal asociación, y dejan entrever la percepción de tres momentos de estas problemáticas, que curiosamente coinciden con diferentes momentos de la historia del barrio. Es decir, en los primeros años de existencia del barrio, éste no contaba con el estigma de peligrosidad que tiene en la actualidad; además, los robos, el consumo y expendio de sustancias psicoactivas eran escasos, al igual que la presencia de manifestaciones de violencia. Algunos habitantes señalaron que ésto se explica con el hecho de que en ese periodo se contaba con presencia permanente de la policía y de entidades estatales y no estatales, las cuales permanecían en el sector realizando un trabajo post-terremoto, lo que permitió la consolidación de redes de apoyo, educación ciudadana y trabajo comunitario.

Posteriormente, este lugar se vio influenciado por procesos migratorios, tanto de personas provenientes de otras ciudades que se movilizaron hasta aquí voluntariamente buscando opciones laborales y de mejoramiento de su calidad de vida, como de personas desplazadas, dejando como resultado que algunos pobladores iniciales migraran del barrio y pusieran en alquiler o comodato sus viviendas. Dicha circunstancia generó un asentamiento de personas con escasas 
entradas económicas y pocas oportunidades laborales, lo cual encrudeció la pobreza y la insatisfacción de necesidades básicas en las familias del sector. Paralelamente, hubo un descenso en la presencia de las organizaciones gubernamentales y no gubernamentales, ocasionando una serie de condiciones que desencadenaron la ocurrencia de situaciones de violencia, delincuencia, y un aumento tanto en el consumo como en el expendio de drogas y en el asentamiento de bandas y pandillas.

Para profundizar y dar explicación a este segundo momento, Esquivia (2006) y Pereira (2008) refieren que tanto el consumo de sustancias como el expendio se encuentran ligados con la mayoría de los acontecimientos y acciones delincuenciales que ocurren en éste, y a su vez, están en estrecha relación dialéctica y compleja con la pobreza, el desempleo y la carencia de redes sociales y de apoyo tendientes a generar una disminución en el capital social.

Entonces, estos tres momentos de la historia del barrio son fundamentales para entender el encrudecimiento de las problemáticas existentes y el nivel crítico al que han llegado la presencia de violencia, consumo y expendio de sustancias, ligadas a la aparición de bandas y pandillas y a la escasa participación social dentro del barrio, lo cual pone de manifiesto la necesidades de implementar programas bienestar social y psicológico y de generación de capital social.

Al estar presentes estos fenómenos de manera constante en el sector, se ha suscitado un tercer momento, que se refiere a una percepción distinta sobre la violencia y la inseguridad por parte de la comunidad, sustentada en las afirmaciones sobre la disminución de ocurrencia de estas problemáticas; sin embargo, lo que habría que preguntarse es si tal situación corresponde en realidad a una solución de estas dificultades, o por el contrario, a un encrudecimiento de las mismas, en tanto se ha pasado de una delincuencia desorganizada a una organizada en torno a bandas dedicadas al control y expendio de drogas.

Para comprender las implicaciones que este fenómeno ha generado en el barrio, es necesario hacer una revisión previa de algunos autores que han abordado esta problemática, analizando cómo inciden sobre las personas, las familias, las comunidades y las sociedades que la padecen.

Así, es preciso referir que las drogas en la sociedad contemporánea constituyen una problemática de salud pública; a finales del siglo pasado en EE.UU. murieron entre 50.000 y 60.000 personas por conflictos relacionados con la provisión de drogas, mientras que los usuarios que murieron debido al consumo abusivo oscilaron entre los 8.000 y 14.000; esta situación es un reflejo de lo que ocurre a nivel mundial en la actual guerra de las drogas. (ONU, 2012)

Otro aspecto ligado a la distribución y el poder sobre las drogas, según Pereira (2008), tiene que ver con la creación de redes de poder que, buscando el monopolio absoluto del negocio, entran en retaliaciones que aportan una innumerable escalada de hechos violentos y de resquebrajamiento del capital social existente dentro de las comunidades, generando miedo, terror y silencio en éstas.

Esta problemática, además de los perjuicios a nivel individual que acarrea, ha afectado significativamente a algunas familias del barrio, puesto que ha generado disfuncionalidad, en la medida que los procesos en torno al manejo de autoridad, comunicación y afecto por parte de los padres, se están viendo comprometidos por 
su poca capacidad de tolerancia y las dificultades en la tarea de educar a sus hijos en la prevención del consumo de sustancias y la enseñanza de valores.

Paradójicamente, a nivel comunitario hay quienes se benefician con la comercialización ilegal de las drogas, tal es el caso de las llamadas bandas, pandillas o grupos de microtráfico. Llama la atención que, si bien algunos habitantes hicieron referencia a este tema, la mayoria de sus intervenciones fueron reticentes, lo cual obedece probablemente al temor que las bandas infunden. Es precisamente esta ambigüedad mostrada en los habitantes acerca de la ausencia de bandas dedicadas a la comercialización de droga, el expendio de la misma y la manifestación de una percepción de disminución de la violencia, la que contrasta con una oleada de graves eventos violentos que se han asociado por parte de las autoridades, lideres, representantes de otras instituciones e incluso de algunos habitantes, a bandas de microtráfico.

Este tercer momento, que podría corresponder al paso de una delincuencia organizada en el barrio en torno al expendio y consumo de drogas. Para entender esta afirmación, antes es preciso describir cómo tales problemáticas, al interrelacionarse entre si, afectan a las personas y a la organización social de las comunidades en donde se presentan. Al respecto, Palacios (2010) afirma:

"los pobladores de las zonas quedan relegados y en medio de la batalla por los espacios de poder que les permita a uno continuar con su negocio y a otros evitarla. Las confrontaciones violentas implican que los espacios comunes han sido invadidos. Los caminos son bloqueados, el plantel del bachillerato es cuartel, entre otros. Hoy lo público se ha tenido que volver privado, se tiene que cuidar de no hablar abiertamente en espacios comunes, se disminuye el tránsito por las carreteras en las noches y así poco a poco ha cambiado la vida cotidiana..." (p.10) y continua diciendo "El momento previo a cualquier exposición frente a actores externos vuelve a la mente inmediatamente una situación de riesgo, queda ahí un miedo latente o al menos una precaución que no se tenía antes." (Palacios, 2010. p.12)

En este orden de ideas, es posible afirmar que la presencia de delincuencia organizada genera una dinámica y organización comunitaria particular, y una vida cotidiana que se caracteriza por una violencia oculta, procesos de justicia por cuenta propia, quebrantamiento de lazos sociales y capital social, cambios en las dinámicas familiares, e instauración del temor y del silencio como defensa ante la violencia.

En síntesis, la precepción de la comunidad de que los hechos delictivos han disminuido no sugiere necesariamente que el expendio y consumo de drogas haya dejado de presentarse, por el contrario, es un fenómeno que sobrevive y se beneficia a partir de la ausencia de redes de capital social que agrupen hacia alternativas distintas a niños, jóvenes y adultos de este barrio. En este sentido Rementería (2009), afirma que el éxito de las drogas radica en que en la actualidad, el contexto social y globalizado se encuentra tranversalizado por la incertidumbre, la precariedad laboral, el desempleo, la exclusión, la intolerancia urbana, la pobreza cultural, el sometimiento político, la farandulización de la participación política y la intolerancia cultural, motivo por el cual estas sustancias mantienen un papel funcional dentro del contexto, ejerciendo el rol de "reconstituyente de almas", almas que buscan asumir un tiempo de reposo y de escape frente al dolor y la ansiedad de la postmodernidad. 
Dicha premisa se ve reflejada en algunos testimonios de habitantes del barrio: "La pobreza es causada por tanto desempleo"; "creo que hay mucho vicio por la ambición de los demás y creo que roban porque les gusta vender esas cosas para comprar vicio y creo que matan porque les pagan para matar" (Tomado de taller con jóvenes).

La transición de una delincuencia desorganizada a una organizada ha generado cambios en la participación social, las relaciones vecinales y han afectado el capital social de la comunidad. Por esta razón, se hace pertinente exponer en un apartado lo evidenciado sobre relaciones vecinales y organización comunitaria, con el fin de analizar los procesos de capital social presentes.

\section{Relaciones vecinales y organización comunitaria.}

En relación con este aspecto, existen diferencias en las percepciones de los habitantes. Los grupos etarios de los niños y los adolescentes reportan la existencia de conflictos entre vecinos, violencia intrafamiliar, robo por parte de habitantes del barrio, expendio y consumo de sustancias, entre otras problemáticas. En el desarrollo de un taller con niños, uno de ellos afirmó: "a mí no me gusta que la gente robe porque consume droga ni que fumen marihuana porque dañan a los otros, fumando se antojan y cuando quieren ya fuman." Esta opinión contrasta con la del grupo etario de los adultos mayores, quienes afirman en general que el barrio es agradable, y que no todo es malo; dado que en éste hay buenas relaciones con los vecinos manifestada en la solidaridad y algunas relaciones de amistad que se pueden tejer en el sector. En este sentido, una adulta mayor del barrio comentó: "el barrio es muy alegre, uno mismo se hace el ambiente" (Tomado de taller con adultos mayores). No obstante, es necesario anotar que algunos adultos mayores también mencionan la existencia de conflictos y tensiones entre los vecinos que han empezado a opacar lo positivo.

Teniendo en cuenta que la percepción de la comunidad en torno a la presencia de relaciones vecinales conflictivas y de diversas manifestaciones de violencia a nivel familiar y comunitario incide directamente en las acciones que sus habitantes realizan por la consolidación de redes de capital social, se contrastarán las problemáticas identificadas anteriormente con dicho concepto, buscando describir cómo está presente cada tipo de capital social en el barrio. Además, se propondrán posibles estrategias de intervención que propenden por la construcción de procesos para potencializar recursos en esta comunidad.

Para Arriagada (2006), el capital social es el conjunto de redes con normas, valores y opiniones compartidas entre grupos de un colectivo humano, los cuales facilitan la cooperación entre dos partes, ya que mide la sociabilidad humana en aquellos aspectos que permiten la colaboración y el uso de recursos por parte de los actores individuales y de las oportunidades que surgen en estas relaciones sociales para llevar a cabo la acción colectiva, de tal manera que el capital social ayuda a la implementación de redes de apoyo para las poblaciones que se encuentran con dificultades que los están afectando.

A partir de este concepto se han descrito tres tipos de redes que ayudan a observar el tipo de lazos y capital social con el que cuenta una comunidad; precisamente el comportamiento de la comunidad del barrio se analizó a la luz de esta descripción. 
Estos tres tipos de redes son: el capital social de unión, el capital social de puente y el capital social de escalera.

El capital social de unión actúa con redes familiares, de amistad cercana y de vecindad y / o comunidad; se caracteriza porque involucra nexos con pocas personas, y en términos geográficos son nexos con personas que viven muy cerca, haciendo que se tornen en redes de alta densidad o fuertes lazos sociales, capaces de llevar a cabo soluciones integradas y productivas para hacer frente a las situaciones problema.

Este tipo de capital se vive en la comunidad, pero no es una generalidad, ya que como lo reportan algunas personas, es posible apoyarse y solicitar favores sólo a algunos vecinos, lo que no sólo está evidenciando la carencia de este tipo de redes, sino el miedo que parece existir en el barrio frente a ciertas circunstancias y hechos de violencia. También se encontró que en el barrio viven personas a quienes no les agrada esta situación y pueden formar redes, de modo que aporten esfuerzos e iniciativas para afrontarla. Un ejemplo de ello lo constituyen las iglesias cristianas, instituciones que de forma frecuente organizan actividades que permiten fortalecer redes sociales en la comunidad. Lo anterior es sugerido por una habitante: "la iglesia es lo que más me gusta del barrio, por el culto y los hermanos que van" (Tomado de taller con adultos).

En relación a esto, es posible encontrar también algunos grupos de jóvenes -especialmente indigenas- quienes han emprendido actividades con el fin de mitigar las problemáticas mencionadas, que terminan encrudeciendo los ciclos de violencia, pobreza, consumo y expendio. Es de destacar una experiencia que adelanta un integrante de la comunidad yanacona, quien de forma particular ha creado una escuela de tradiciones y saberes propios de su grupo étnico que funciona los fines de semana y cuyos beneficiarios son los niños yanaconas del sector.

En cuanto a la segunda forma de redes, el capital social de puente, éste se encarga de redes organizacionales, juntas vecinales de comunidades de campesinos, de madres solteras, entre otras; se caracteriza por las formas federativas de organización entre grupos similares.

En el barrio sobresale la presencia de la Junta de Accion Comunal (JAC), organización social y cívica presente de forma permanente que adelanta procesos de unión comunitaria a través de diversos comités, como el de adulto mayor, trabajo, medio ambiente y conciliación; es de resaltar el andamiaje brindado a la población de adultos mayores, que hoy se considera como una agrupación fuerte en el barrio, en la que sus miembros han establecido lazos afectivos fuertes entre sí. Es pertinente sugerir la conformación en el barrio de una red que agrupe a los adolescentes; es bien sabido que hay algunos jóvenes que actualmente pertenecen a bandas delictivas y son agentes activos en el microtráfico y en el consumo de sustancias, pero también hay quienes rechazan estas actividades y se resisten a ser involucrados, lo cual prioriza la importancia de que los jóvenes conformen y construyan juntos un capital social de puente que actualmente no existe, a partir del diálogo sobre sus problemáticas, de la manifestación sobre la convicción de algunos de no elegir esas opciones y de la movilización hacia acciones que contribuyan a hacer mejor el ambiente en su barrio. La creación y consolidación de esta red de adolescentes es una propuesta de estrategia de intervención, que puede ser facilitada por equipos psicosociales de entidades gubernamentales o de 
carácter privado, a través de proyectos educativos, que les permitan encontrar un sentido de vida y crecer a través del aprendizaje de un oficio, un arte o un deporte.

La tercera forma de red es el capital social de escalera, relacionada con las redes de grupos y personas de diferente identidad y grados de poder sociopolítico; se caracteriza por los nexos que crean relaciones medianamente consolidadas entre personas y/o comunidades, agencias públicas o no gubernamentales externas que facilitan el acceso a esferas políticas, a recursos de agencias externas y al apoyo de estas agencias en momentos de crisis o amenaza.

Esta modalidad de capital social está presente en el barrio, pero con algunas falencias. Por parte de la Gobernación, la Alcaldía y otras instituciones gubernamentales como ICBF, Policía y SENA e instituciones no gubernamentales como Visión Mundial, Fundación Telefónica, Pro-niño, se realizan brigadas de salud, charlas, y otras actividades de tipo psicosocial, pero ninguna de estas organizaciones ha logrado consolidar una red comunitaria cohesionada y empoderada.

Lo anterior puede deberse a que los habitantes del sector ya no sienten interés por participar en estas actividades, en tanto no hay presencia constante de estas instituciones, pues generalmente ejecutan proyectos de corta duración, sin una continuidad. Otro elemento que puede estar incidiendo en este proceso es la inexistencia de sedes o infraestructura propia, que imposibilita procesos de legitimidad y confianza en los procesos y obstaculiza el desarrollo de un trabajo continuo y permanente. En este sentido, se hace imprescindible la adecuación de estos espacios físicos, como una casa de justicia, un centro de salud y un centro de desarrollo comunitario donde puedan llevarse a cabo programas de educación y acompañamiento psicosocial dirigidos a habitantes de todos los grupos etarios.

Adicionalmente, se sugiere que las estrategias planteadas desde cualquier institución tengan continuidad, con el objeto de que la comunidad perciba su presencia y empiece a reconocer a las instituciones como líderes y como movilizadoras de diferentes capitales sociales.

Enseguida se hablara del concepto de bienestar social, y a la luz de estas consideraciones teóricas, se contrastará con la situación del barrio. La definición del bienestar supone un reto complejo y ambiguo, pues remite a preguntas de carácter subjetivo relacionadas con los conceptos de felicidad y satisfacción; se han realizado diversas investigaciones sobre este tema, las primeras se centraron en correlacionar el bienestar con variables como la edad, el sexo, los ingresos, la salud, etc. En la actualidad, las investigaciones se centran en la comprensión de los procesos que subyacen al bienestar, al estudiar cómo cada una de esas experiencias, y las condiciones sociales facilitan o dificultan su consecución.

Ryff (1997), propone una perspectiva diferente del bienestar psicológico, en la que tiene en cuenta aspectos como contar con un propósito en la vida y esforzarse por afrontar las dificultades que se presenten y por conseguir las metas propuestas; en cuanto al bienestar subjetivo, lo vincula con sentimientos de relajación, ausencia de problemas y presencia de sensaciones positivas.

Al dar una mirada a la vida de algunos jóvenes del barrio, se encuentra un panorama alarmante, puesto que su propósito de vida está orientado hacia el consumo de sustancias y la participación en actividades delictivas. La comunidad aludió que 
las causas son la falta de alternativas para ocupar su tiempo y de oportunidades laborales, pero más allá de estos factores, puede afirmarse que en los adolescentes carecen de un propósito vital, de una voluntad de sentido, es decir, que se dirijan con sus ideas, sentimientos y acciones hacia un sentido que primeramente deben descubrir y cuya plenitud deben lograr.

Se necesitan escuelas de formación artística, deportiva, y de diversos oficios, pero para facilitar estos procesos formativos, los jóvenes deben conocer primero posibles objetivos de vida, las alternativas que existen para elegir ser alguien, y entender que deben tomar una decisión responsable, y movilizarse a través de acciones para mejorar su bienestar psicológico, y a su vez, influir positivamente en el bienestar social de la comunidad.

De acuerdo a Keyes \& Shapiro (2006), "desde la perspectiva de la salud, un individuo debe ser considerado sano sí dispone de altos niveles de bienestar social, por ejemplo, si se siente bien integrado en su comunidad" (Blanco, Díaz 2006, p. 12). Adicionalmente, Keyes afirma que la salud social positiva se encuentra relacionada con las diversas áreas de la vida social, de modo que son más saludables aquellas personas que profesan un arraigado sentimiento de pertenencia y cuentan con vínculos sociales fuertes como apoyo; también quienes confian en los demás y en sí mismos, y se aceptan tal como son, viviendo sin dramatismos los aspectos positivos y negativos de su vida; igualmente las personas que guian su vida sin prestar mayor atención a las presiones y/o convenciones sociales; quienes se perciban como útiles a la colectividad, creen en el futuro de la sociedad, admiten su potencial de crecimiento y la posibilidad de obtener provecho de él; y finalmente, las personas que ven su vida y el mundo como algo lleno de sentido y en dirección a un propósito. (Blanco, Díaz, 2006)

Al hacer una revisión de la comunidad de Las Colinas, y teniendo en cuenta las áreas anteriormente enunciadas, se puede evidenciar que el nivel de bienestar social no es el óptimo, en tanto que la comunidad en general manifiesta sentirse a gusto en el barrio, pero no manifiestan un sentimiento de pertenencia fuerte hacia él. Esto se refleja en el inadecuado manejo que dan a las basuras y residuos sólidos, y en la poca participación en actividades de cuidado y de reflexión sobre las problemáticas y soluciones. El presidente de la Junta de Acción Comunal del barrio afirmó en este sentido: "Yo me preguntó, no hay conciencia con el tema ambiental, arrojamos las basuras en el sitio que nos dé la gana. Igualmente, cuando se organizó una jornada de amor por el barrio, que se limpiaron algunas calles, casi no hubo colaboración, pero sí salian a preguntar si les íbamos a limpiar el frente de la casa". (Tomado de reunión con líderes comunitarios).

Además, la presencia de personas dedicadas a actividades delictivas y los procesos de temor y disipación en la comunidad que éstos han generado, han favorecido que la ausencia de empatía hacia los demás y las acciones que violan la ley se hayan incrementado. En esta medida, es una realidad y una forma de vida que actualmente aprenden y reproducen niños y jóvenes del barrio; lo cual es grave y sugiere otra falencia a nivel comunitario, que se relaciona con sentirse útil a la colectividad y creer en el futuro de la sociedad. 


\section{Conclusiones.}

A partir del análisis de los resultados, se puede concluir que uno de los factores que favoreció la generación de las múltiples problemáticas que se presentan en el barrio fue la deficiente planeación y ejecución de su construcción, que se derivó en la creación de un barrio con sólo tres manzanas, una sola vía de acceso, y ausencia de zonas verdes, espacios deportivos, culturales y de un centro de salud. Su localización geográfica, junto con los múltiples hechos delictivos que han sucedido en los últimos años han generado que esta comunidad atraviese por un proceso de segregación urbana; un hecho que, a su vez, hace posible afirmar que el barrio se encuentra atravesando por procesos de exclusión social y pobreza urbana.

Dentro de las concepciones actuales sobre exclusión social, es posible hablar de ésta no sólo cuando se carece de oportunidades o de inclusión, sino que como lo refiere Perona (2010), el termino exclusión "tiene mayor potencialidad analítica para referirlo a aquellas situaciones que implican "fuerte acumulación de desventajas" (p. 15). Es asi como ante la poca presencia estatal, la carencia de programas que acentúen la posibilidad de generación de capital social, y ante los hechos generalizados de violencia, es posible hablar de una acumulación de desventajas para la población que termina por sumirlos en la desigualdad, la precariedad y el miedo.

Si bien gran parte de los habitantes del barrio cuentan con servicios públicos y son propietarios de su vivienda, ésto no hace que la pobreza haya desaparecido de sus vidas y que la carencia en otros aspectos no ronde su cotidianidad; una evidencia de ello es que los espacios comunitarios, educativos, deportivos, culturales y de salud son sumamente escasos.

Otra conclusión que se deriva de este diagnóstico es que el cambio en la percepción de la comunidad sobre la violencia e inseguridad en el barrio, que indican el supuesto descenso en la ocurrencia de estas problemáticas, probablemente permite inferir que se ha pasado de una delincuencia desorganizada a una organizada en torno a bandas dedicadas al control y expendio de drogas. (Buscaglia et al., 2002). Así, la presencia de delincuencia organizada ha generado una dinámica comunitaria particular y una vida cotidiana que se caracteriza por situaciones de violencia oculta, procesos de justicia por cuenta propia, quebrantamiento de lazos sociales y capital social, cambios en las dinámicas familiares, e instauración del temor y del silencio como defensa ante la violencia. 


\section{Referencias bibliográficas.}

Aguilar, M. Ander-Egg, E. (2001). Diagnóstico social: Conceptos y metodología. Argentina Mexico: Lumen, Hvmanitas. Recuperado de http://carmonje. wikispaces.com/file/view/DIAGNOSTICO+SOCIAL-+Mar\%C3\%ADa+Jos\%C3 \%A9+Aguilar+y+Ezequiel+Ander+Egg.pdf.

Alberich, T. (2007). Investigación-Acción Participativa y mapas sociales. Recuperado de https://www.uji.es/bin/serveis/sasc/ext-uni/oferim/forma/jorn/tall.pdf

Arriagada, I. (2006) Breve guía para la aplicación del enfoque de capital social en los programas de pobreza. Chile: CEPAL.

Baró, I. (2004). Acción e ideología: psicología social desde Centroamérica. España: UCA editores.

Bernabéu, J. Casullo, M. González, R. \& Montoya, I. (2002). Relación entre estilos $\mathrm{y}$ estrategias de afrontamiento y bienestar psicológico en adolescentes. Psicothema, Volumen 14 (2), 363-368. Recuperado de file:/ / C:/Users/paula/ Downloads/8028-13731-1-PB.pdf.

Blanco, A. y Díaz, D. (2006) Orden social y salud mental: una aproximación desde el Bienestar social. Ciencia y Salud. 17 (1): 7-29.

Blanco, A.Valera, S. (2007). Fundamentos dela Intervención psicosocial. Intervención psicosocial. Prentice Hall. 2 - 57.

Buscaglia, E., González, S., Fumarulo, S., \& Prieto, C. (2002). Delincuencia Organizada y Terrorismo. Su Combate A Través De La Convención De Palermo. Revista Universitaria de la Universidad Católica de Chile. 1 (1), 1-9. Recuperado de http://papers.ssrn.com/sol3/papers.cfm?abstract_id=946.

Concha, A. (2002). Impacto social y económico de la violencia en las Américas. Biomédica, 22(Su2) 347-361. Recuperado de http://estudiosterritoriales.org/ articulo.oa?id=84309604

Gil, J, Pastor, J, De Paz, F, Barbosa, M, Macías, J, Maniega, M, Rami, L, Boget, T, Picornell, (2002). Psicobiologia de las conductas agresivas. Anales de psicología. Volumen 18 (2), 293-303.

Lunecke, A. Ruiz, J. (2006). Capital Social Y Violencia: Análisis Para La Intervención En Barrios Urbanos Críticos. Santiago: Instituto de Sociología.

Pontificia Universidad Católica de Chile. Recuperado de https://www. google. $\mathrm{com} / \mathrm{t} \& \mathrm{rct}=\mathrm{j} \& \mathrm{q}=\& \mathrm{esrc}=\mathrm{s} \&$ source $=\mathrm{web} \& \mathrm{~cd}=1 \& \mathrm{ved}=0 \mathrm{CCkQFjAA} \& u r$ 1=http $\% 3 \mathrm{~A} \% 2 \mathrm{~F} \% 2 \mathrm{Fwww}$. flacsoandes.org\%2Fbiblio\%2Fcatalog\%2FresGet. AFQjCNFqHSV16xgcwZOnxePuG8flgScpYw\&sig2=VzN1uTde0gGkaTm2JAe9cA

La Cronica del Quindío: Más de 800 casos de dengue en Armenia, en lo corrido de 2013. (2013, 26 de Octubre) Recuperado de http://www.cronicadelquindio. com/noticia-completa-titulo-mas_de_800_casos_de_dengue_en_armenia_ en_lo_corrido_de_2013- seccion-armenia-nota-66268.htm. 
Meneses, L. (2013, 03 de Octubre). Por amenazas cancelaron clases en la I.E. Las Colinas. LaCrónicadelQuindio.Recuperadodehttp:/ / www.cronicadelquindio. com/noticia-completa-titulo-por_amenazas_cancelaron_clases_en_la_i_e_ las_colinas-seccion-armenia-nota-65531.htm.

Ministerio de Sanidad y Consumo. (2007). Guía Sobre Drogas, delegación del gobierno para el plan nacional sobre drogas. España. Recuperado de http:// www.pnsd.msc.es/Categoria2/publica/pdf/guiaDrogas.pdf.

Monserrat, A. Muñoz, $\mathrm{M}^{\mathrm{a}}$.(2003). Aspectos psicosociales de la violencia juvenil. Revista Estudios de Juventud. 62 (1), 49 - 134. Recuperado de http://www. injuve.es/sites/default/files/62completa.pdf.

Organización Mundial de la Salud (2004). Invertir en salud mental. Ginebra. Recuperado de http://www.who.int/mental_health/advocacy/en/ spanish_final.pdf.

Moscovivi, S. (1986). Psicología Social. España: Paidós.

Organización Mundial de la Salud (2006). Manual de recursos de la OMS sobre salud mental, derechos humanos y legislación. Recuperado de http:/ /apps.who.int/iris/bitstream/10665/43478/1/9243562827_spa. pdf.

Organización Mundial de la Salud (2001). Informe de la salud mental en el mundo.

Recuperado de http://whqlibdoc.who.int/whr/2001/WHR_2001_spa.pdf.

Organización Naciones Unidas. (2012). Informe mundial sobre las drogas 2012. Naciones Unidas. Oficina contra la droga y el delito. Recuperado de http:// www.unodc.org/documents / data-and-analysis /WDR2012/WDR_2012_ Spanish_web.pdf.

Palacios, E. (2010). Implicaciones en una localidad rural del enfrentamiento armado entre el gobierno de México y la delincuencia organizada. Porik An Revista facultad de ciencias contables, económicas y administrativas Universidad del Cauca. 12 (15). 9-32.

Pereira, M. (2008). "Pobreza y exclusión en las favelas de Río de Janeiro". (pp. 213247) En: Ziccardi, A. (2008) (ed). Procesos de urbanización de la pobreza y nuevas formas de exclusión social. Los retos de las politicas sociales de las ciudades latinoamericanas del siglo XXI. P 420. Bogotá: Siglo del Hombre Editores, Clacso-Cropp. Recuperado de http://bibliotecavirtual.clacso.org. ar/clacso/clacso-crop/20120504120512/ziccardi.pdf.

Perona, N. (2001). Desde la marginalidad a la exclusión social. Una revisión de los conceptos. La Sociología en sus Escenarios, centro de estudios de opinión. 5, 1 - 18. Recuperado de http://aprendeenlinea.udea.edu.co/revistas/index. $\mathrm{php/ceo/article/viewFile/7479/6886.}$ 
Pórtela, D. (2006) El Volcán: Etnografia de un ghetto en Santiago" Identidad, capital social y control cultural en la Vivienda Social. (Tesis para optar al grado de Licenciado en Antropología Social).Universidad Academia De Humanismo Cristiano Escuela De Antropologia, Santiago de Chile. Recuperado de http://bibliotecadigital.academia.cl/bitstream/123456789/998/1/tant51.pdf.

Rementería, I. (2009 Julio - Agosto). La guerra de las drogas: cien años de crueldad y fracasos sanitarios. Revista nueva sociedad. $\mathrm{N}^{\circ} 222$. Recuperado de http:// www.nuso.org/upload/articulos/3622_1.pdf.

Rojas, G. (2011, 28 de Agosto). Maestros en taller de Derechos Humanos. La Crónica del Quindío. Recuperado de http://www.cronicadelquindio.com/ noticia-completa-titulo-maestros_en_taller_de_derechos_humanos-secciongeneral-nota-35186.htm.

Ruiz, J. (2008). Violencias En Barrios Críticos En Santiago: Narcotráfico y transformación del capital social en la periferia simbólica de la ciudad. (Tesis para optar al grado académico de Magíster en Desarrollo Urbano). Instituto de Estudios Urbanos Territoriales de la Pontificia Universidad Católica de Chile. Recuperado de http://www.estudiosurbanos.uc.cl/ wp-content/uploads / $2011 / 01$ /ViolenciaenbarrioscrticosenSantiago. Narcotrficocapitalsocialyp eriferiasimblica.pdf.

Sepúlveda, L. (2014, 28 de Abril). S.O.S por cuatro barrios del sur de Armenia; Observatorio de la Uniquidío prendió alarmas por focos por delincuencia. El Tiempo. Recuperado de http://www.eltiempo.com/colombia/eje-cafetero/ ARTICULO-WEB- NEW_NOTA_INTERIOR-13894975.html.

Velasquez, C. (2010). Medellín desconectada... de la dignidad, Javilando, 2 (1) 103 $-109$.

Velasquez, C. (2009). Situación actual en la presentación de los servicios públicos. Medellin. El Agora USB, 9 (2), 391-425.

Velasquez, C. (2012). La politica de mejoramiento integral del barrio en Medellín ¿reconquista del territorio por parte del Estado? Kavilando, 3 (2), 72-78.

Ziccardi, A. (2008). Procesos de urbanización de la pobreza y nuevas formas de exclusión social. Los retos de las politicas sociales de las ciudades latinoamericanas del siglo XXI. Bogotá: Siglo del Hombre Editores, ClacsoCrop. Recuperado de http://bibliotecavirtual.clacso.org.ar/clacso/clacsocrop/20120504120512/ziccardi.pdf 
Nota.

1 Agradecemos también al trabajo arduo, constante y decidido de los psicólogos egresados de la Universidad de San Buenaventura Medellín extensión Armenia, que hicieron parte de esta investigación: Jhon Alexander Sánchez, Maria Fernanda Calderón, Ximena Marín, Leonardo Valencia y Deisy Viviana Parra. 
